\title{
An optimal placement of phasor measurement unit using new sensitivity indices
}

\author{
K. Khalid, A. A. Ibrahim, N. A. M. Kamari, M. H. M. Zaman \\ Department of Electrical, Electronic and Systems Engineering, Faculty of Engineering and Built Environment, \\ Universiti Kebangsaan Malaysia, Malaysia
}

\begin{abstract}
Article Info
Article history:

Received Mar 24, 2020

Revised Jun 2, 2020

Accepted Jul 15, 2020

\section{Keywords:}

Integer linear programming Observability contraints Phasor measurement unit Power system stability

Zero injection bus

ABSTRACT

This paper presents an alternative approach to solve an optimal phasor measurement unit (PMU) placement by introducing two new sensitivity indices. The indices are constructed from the information measured from PMUs such as voltage magnitude and angle. These two parameters are essential for power system stability assessment and control. Therefore, fault analysis is carried out to obtain the voltage magnitude and angle deviations at all buses in order to construct the indices. An exhaustive search method is used to solve the linear integer programming problem where all possible combinations of PMU placement are evaluated to obtain the optimal solution. Unfortunately, the traditional objective function to minimize the total number of PMU placement leads to multiple solutions. The indices can be used to assess multiple solutions of PMU placement from the exhaustive method. In this work, an optimal solution is selected based on the performance of PMU placement in according to the indices. The proposed method is tested on the IEEE 14 bus test system. Only four PMUs are required to monitor the whole test system. However, the number of PMUs can be reduced to three PMUs after applying zero injection bus elimination.
\end{abstract}

This is an open access article under the CC BY-SA license.

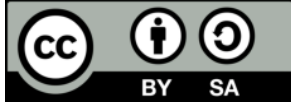

\section{Corresponding Author:}

A. A. Ibrahim,

Department of Electrical, Electronic and Systems Engineering,

Faculty of Engineering and Built Environment,

Universiti Kebangsaan Malaysia, 43600 Bangi, Selangor, Malaysia.

Email: ahmadasrul@ukm.edu.my

\section{INTRODUCTION}

Power system failures and fault occurrence are unpredictable due to the revolution of complex network growth and uncertain nature catastrophic. This leads to insecure and unstable operation of the power system [1]. Therefore, a wide area monitoring scheme is extremely important to monitor the whole power system operation and make a proper preparation for possible system stability issues [2-7]. Phasor measurement unit (PMU) is installed for this monitoring purposes. The device measures synchronized data of voltage phasor, current, and power that can be used to accurately estimate the system condition [8,9]. The advantageous of PMU installation that enable real-time measurement of voltage, current and frequency have provided attracted many reearchers to study its applications in power system. One of the applications that can be used from the PMU information is a power system stability assessment [10-12]. Although the PMUs provide various solution in solving power system issues, the installation cost is still expensive and accumulate of repeated data acquisition could cause overload in the monitoring system [13]. As a solution, a precise management in PMU placement is required. 
Over the past years, several works have been carried out to determine an optimal PMU placement (OPP) while maintaining full observability of power networks by using redundancy index to select the best PMU placement [14-19]. The redundancy index in OPP is used as indicator to decide the best combination from several feasible PMU placement solutions. However, the redundancy index might not give enough information for power stability assessment. In this work, an alternative method to select the best solution is suggested by using new indices that consider potential parameters for system stability assessment. All possible combinations will be evaluated using the indices and they are ranked according to their scores. The number of evaluations can be reduced by applying certain electrical rules such as ohm's law and zero injection bus (ZIB) [20, 21]. The ZIB is defined as a bus without any load or generator. So, the ZIB can be ignored because it doesn't have any power injections to give effects on electrical parameters.

A remedial action for mitigating power system failures and cascade power outages could not be taken without a proper monitoring system. Therefore, a suitable location of PMU placement is essential to monitor the whole system for system prevention and restoration. However, the number and location of the PMUs dependin on fault occurrence [13, 22-24]. Normally, the OPP problem is solved using connectivity matrix without considering any restrictions from the network operation. The fault events will affect the operating network parameters such as voltage magnitudes and angles. Thus, they are important to be considered for the PMU placement that can be used for remedial actions in preventing any power interupptions [25]. In this work, voltage magnitude and angle are considered as new benchmark indices to solve the OPP problem.

\section{OPTIMAL PMU PLACEMENT FORMULATION}

An optimal PMU placement is formulated based on linear integer programming problem. An exhaustive search is carried out to solve the problem where all possible combinations of PMU placement are evaluated to identify the minimum number of PMU placement while maintaining full observability of power networks [19]. In the optimal PMU placement formulation, certain rules are considered as stated in [26]:

a. PMU installed at a certain bus will able to observe all adjacent buses that are connected to it. The branch current phasor of all lines connected to the bus can be obtained. This is known as direct measurement.

b. Voltage phasor of the adjacent buses can be calculated from line impedance as the voltage and current phasor at one end is known.

c. If the voltage phasors at both ends of a transmission line are known, the current flow through the line can be calculated from the line impedance. This is known as pseudo-measurement. By applying the mentioned rules in finding the OPP with ineger linear programming approach multiple possible solution will be obtained. PMU placement is modelled as binary variable $x_{i}$, as expressed below,

$$
x_{i=}\left\{\begin{array}{l}
1, \text { if a PMU is isnstalled at bus } i \\
0, \text { otherwise }
\end{array}\right.
$$

According to (1), the total number of PMU placement can be calculated from a summation of the binary variable of placement, $x$. Therefore, the objective to minimize the total number of PMU placement can be described as:

$$
\min \sum_{i=1}^{N} x_{i}
$$

that subjects to full system observability constraints to ensure the whole system network is monitored. This can be defined as below:

$$
\text { A. } x \geq w
$$

where, $N$ is total number of bus, $\mathrm{A}$ is a binary connectivity matrix of the network and $\mathrm{w}$ is a column vector of observability control parameter. The observability control parameter is used to set the number of PMUs that will monitor at each bus.

More explaination on determining optimal PMU placement is given based on a five bus system as illustrated in Figure 1. The binary connectivity matrix, A can be obtained from branch data of the network as expressed in (4). Alternatively, it can be directly constructed from the admittance matrix in which all non-zero elements are assumed $1 \mathrm{~s}$ and $0 \mathrm{~s}$. 


$$
a_{i, j}=\left\{\begin{array}{l}
1, \text { if } i=j \\
1, \text { if the bus } i \text { is connected to bus } j \\
0, \text { otherwise }
\end{array}\right.
$$

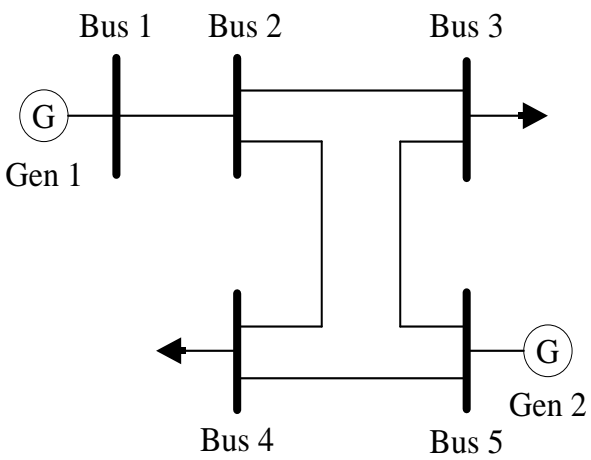

Figure 1. An example of 5-bus system

The binary connectivity matrix A for the 5-bus system can be constructed,

$$
\mathrm{A}=\left[\begin{array}{lllll}
1 & 1 & 0 & 0 & 0 \\
1 & 1 & 1 & 1 & 0 \\
0 & 1 & 1 & 0 & 1 \\
0 & 1 & 0 & 1 & 1 \\
0 & 0 & 1 & 1 & 1
\end{array}\right]
$$

The length of the binary variable of placement, $x$ is equal to the number of bus in the system as given in (6).

$$
x=\left[\begin{array}{lllll}
x_{1} & x_{2} & x_{3} & x_{4} & x_{5}
\end{array}\right]
$$

In (3) can be further expanded by considering $(5,6)$. It can be expreesed as,

$$
\text { A. } x= \begin{cases}f_{1}=x_{1}+x_{2} & \geq 1 \\ f_{2}=x_{1}+x_{2}+x_{3}+x_{4} & \geq 1 \\ f_{3}=x_{2}+x_{3}+x_{5} & \geq 1 \\ f_{4}=x_{2}+x_{4}+x_{5} & \geq 1 \\ f_{5}=x_{3}+x_{4}+x_{5} & \geq 1\end{cases}
$$

According to (7), full observability can be achieved when all elements are equal or greater than one. For example, bus 1 is observed when at least one of the allocated PMUs is installed either at bus 1 or 2 . This clearly shows that size of the problem is propotional to the number of available location for PMU placement. The number of location can be reduced by using the ZIB concept. The ZIB is referred to a bus without any generator or load connected to it. Referring to Figure 1, only bus 2 can be considered as ZIB because there is no generator or load connected to this bus. Therefore, bus 2 can be eliminated all its adjacent buses need to be directly connected to each other to maintain the connectivity. The ZIB elimination process can be done using topology transformation technique. The topology transformation technique is used because it is easy to implement and faster to form a new connectivity matrix, $A_{\text {new. }}$. In this case, buses 1,3 and 4 can be directly connected after removing bus 2 (i.e., the ZIB) and new constraints are formed using union operator as expressed in (8). As a result, total number of constraints can be reduced as shown in (9) before OPP is carried out.

$$
\begin{gathered}
f_{1}^{\text {new }}=f_{1} \cup f_{2}, f_{3}^{\text {new }}=f_{2} \cup f_{3}, f_{4}^{\text {new }}=f_{2} \cup f_{4} \\
\mathrm{~A}_{\text {new }} \cdot x= \begin{cases}f_{1}^{\text {new }}=x_{1}+x_{2}+x_{3}+x_{4} & \geq 1 \\
f_{3}^{\text {new }}=x_{1}+x_{2}+x_{3}+x_{4}+x_{5} & \geq 1 \\
f_{4}^{\text {new }}=x_{1}+x_{2}+x_{3}+x_{4}+x_{5} & \geq 1 \\
f_{5}=x_{3}+x_{4}+x_{5} & \geq 1\end{cases}
\end{gathered}
$$




\subsection{Sensitivity indices for $P M U$ placement}

Voltage magnitude and angle from PMU measurement are the essential information for power stability assessment. Since these variables are commonly used in stability assessment, they can be used as indices to rank and decide the best combination of PMU placement. The indices can be constructed using fault analysis and used to indicate the critical buses in power networks so that priority for PMU installation will be given accordingly. Faults at transmission lines are the prominent issues in the power system [26] and therefore, it is used in this analysis. Fault at $50 \%$ of the transmission lines are considered and carried out using DIgSILENT PowerFactory simulation software. Then, voltage magnitude and angle at each bus for all fault locations are recorded. Two indices based on the voltage magnitude and angle can be constructed by using standard deviation formula as expressed in $(10,11)$, respectively.

$$
\begin{gathered}
\sigma_{i}^{v}=\sqrt{\frac{\sum\left(\Delta v_{i}-\mu_{i}\right)^{2}}{N_{F}}} \\
\sigma_{i}^{\delta}=\sqrt{\frac{\sum\left(\Delta \delta_{i}-\mu_{i}\right)^{2}}{N_{F}}}
\end{gathered}
$$

where, $\sigma_{i}^{v}$ is the standard deviation of voltage magnitude at bus $i$ and $\sigma_{i}^{\delta}$ refers to the standard deviation of the angle. $\Delta v_{i}$ and $\Delta \delta_{i}$ are the different between pre-fault and post fault at bus $i$ for voltage magnitude and angle, respectively. $\mu_{i}$ is the average value of fault data at bus $i$ and $N_{F}$ is the total number of fault locations. The performance indices from the fault analysis are obtained for a specific location. To evaluate the performance of PMU placement, all values of the indices in corresponding to the PMU locations should be taken into account. Therefore, the best solution is selected based on total value of the performance indices depending on the PMU placement as the following:

$$
\begin{aligned}
& \mathrm{SOI} v=\sum_{i=1}^{N}\left(x_{i} \cdot \sigma_{i}^{v}\right) \\
& \mathrm{SOI} \delta=\sum_{i=1}^{N}\left(x_{i} \cdot \sigma_{i}^{\delta}\right)
\end{aligned}
$$

where, $\mathrm{SOI} v$ is a summation of the index based on voltage magnitude and SOI $\delta$ is a summation of the index based on voltage angle. An overall process for the proposed OPP is depicted in Figure 2.

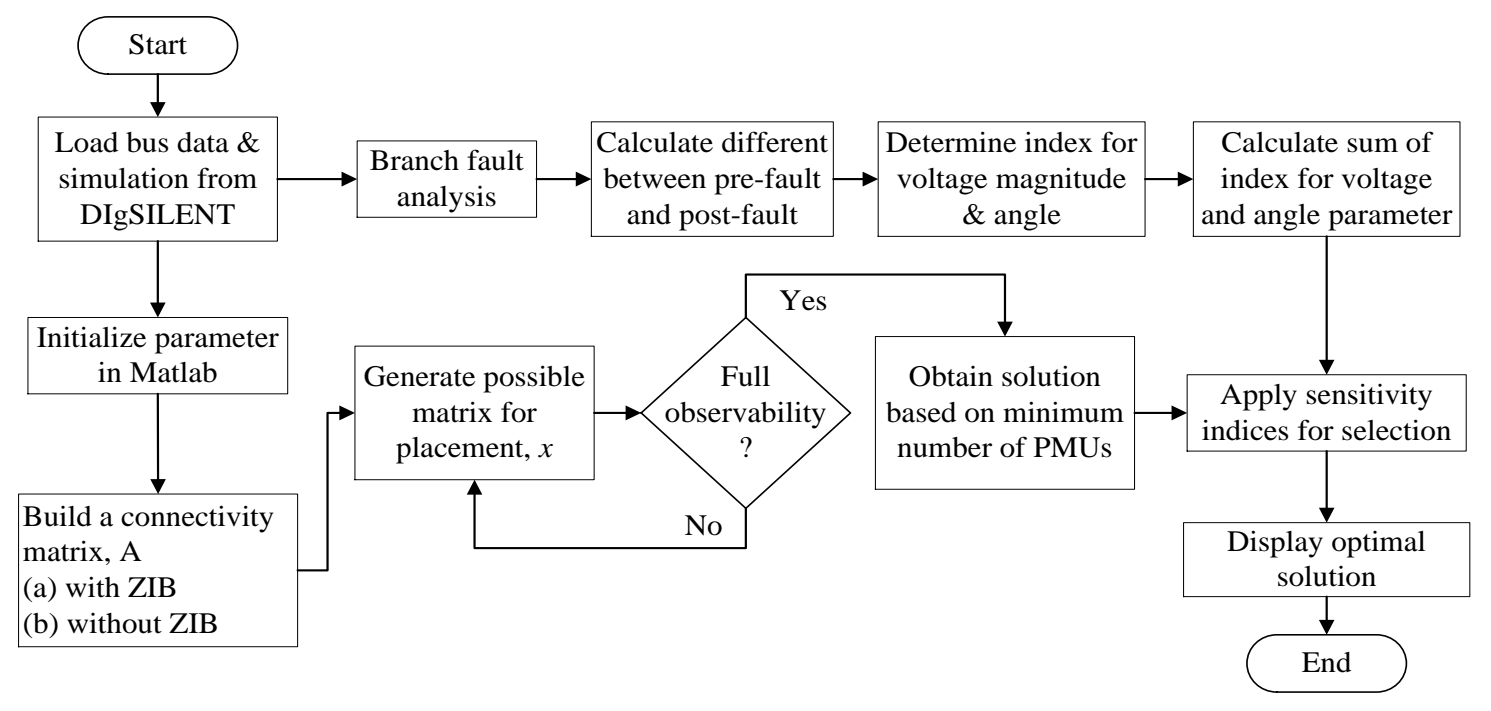

Figure 2. Flowchart for the proposed method

\section{RESULTS AND DISCUSSION}

The proposed OPP has been implemented on IEEE 14-bus test system as depicted in Figure 3. Referring to this figure, the IEEE 14-bus test system contains 5 synchronous generators, a two-winding transformer and a three-winding transformer. One ZIB in the test system is an internal bus of the three-winding transformer (bus 7). The problem is solved within MATLAB environment using 
the exhaustive search method to find the minimum number of PMU installation while maintaining full observability of the power network. The results give 5 possible solutions of PMU placement combinations without considering ZIB elimination and one solution when considering ZIB elimination. The feasible combinations of PMU placement for both cases are given in Table 1 and 2, respectively. Since only one solution after ZIB elimination as shown in Table 2, the selection using the proposed indices is not relevant. Meanwhile, Table 1 shows 5 different solutions at minimum number of PMU placement that able to monitor the whole system. Consequently, a further step is required to identify the best solution.

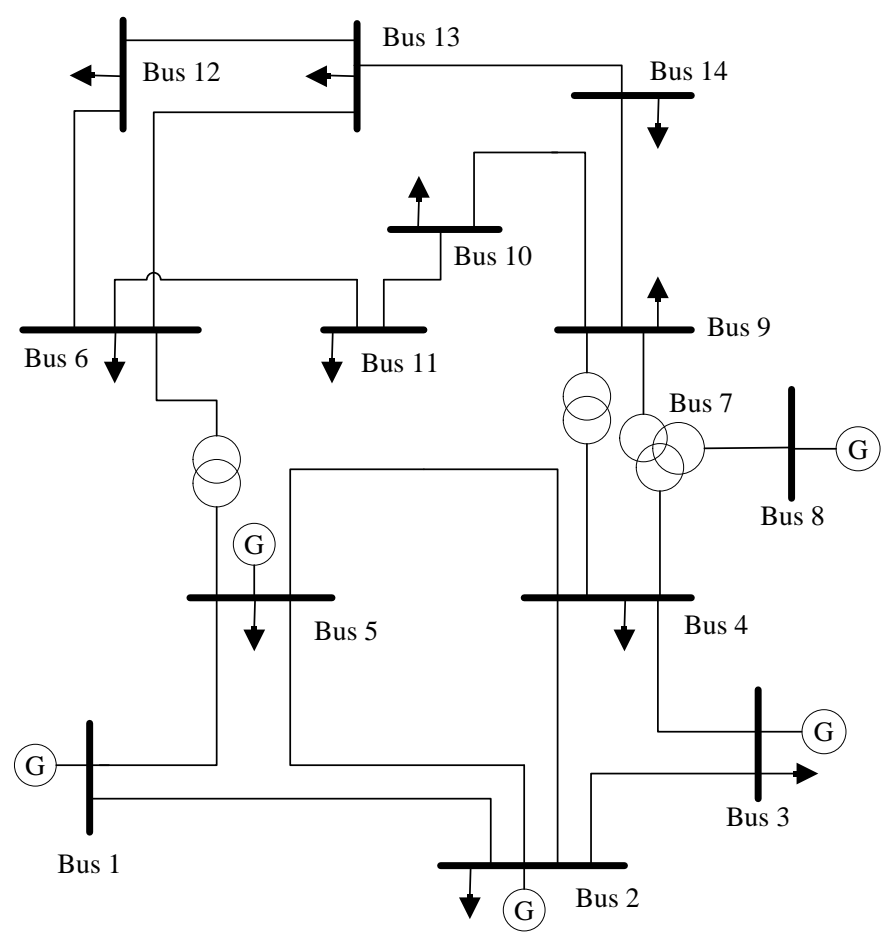

Figure 3. IEEE 14 bus test system

Table 1. OPP multiple solution without ZIB elimination

\begin{tabular}{|c|c|c|c|c|}
\hline Binary combination for PMU placement & \multicolumn{4}{|c|}{ Bus location } \\
\hline$\left[\begin{array}{llllllllllllll}0 & 1 & 0 & 0 & 0 & 1 & 1 & 0 & 1 & 0 & 0 & 0 & 0 & 0\end{array}\right]$ & 2 & 6 & 7 & 9 \\
\hline$\left[\begin{array}{lllllllllllllll}0 & 1 & 0 & 0 & 0 & 1 & 0 & 1 & 1 & 0 & 0 & 0 & 0 & 0\end{array}\right]$ & 2 & 6 & 8 & 9 \\
\hline$\left[\begin{array}{llllllllllllll}0 & 1 & 0 & 0 & 0 & 0 & 1 & 0 & 0 & 1 & 0 & 0 & 1 & 0\end{array}\right]$ & 2 & 7 & 10 & 13 \\
\hline$\left[\begin{array}{llllllllllllll}0 & 1 & 0 & 0 & 0 & 0 & 1 & 0 & 0 & 0 & 1 & 0 & 1 & 0\end{array}\right]$ & 2 & 7 & 11 & 13 \\
\hline$\left[\begin{array}{lllllllllllllll}0 & 1 & 0 & 0 & 0 & 0 & 0 & 1 & 0 & 1 & 0 & 0 & 1 & 0\end{array}\right]$ & 2 & 8 & 10 & 13 \\
\hline
\end{tabular}

Table 2. OPP solution with ZIB elemination

\begin{tabular}{|c|c|}
\hline ent & Bus locatior \\
\hline$\left[\begin{array}{llllllllllllll}0 & 1 & 0 & 0 & 0 & 1 & 0 & 0 & 1 & 0 & 0 & 0 & 0 & 0\end{array}\right]$ & \\
\hline
\end{tabular}

As mentioned earlier, the proposed indices are based on voltage magnitude and angle deviations depending on their locations. Figure 4 illustrates the effects sensitivity levels of each location (bus) in the IEEE 14 bus system toward fault occurance in terms of voltage magnitude and angle. In this figure, the most sensitive bus is represented by the darkest colour with respect to all fault occurences on lines in the test system and vice versa. In Figure 4 (a), buses 1, 2, 3 and 8 can be considered as the least sensitive buses in terms of voltage magnitude because they are merely affected to the fault occurences at branches other than directly connected to them. On the other hand, Figure 4 (b) illustrates the bus behaviours in terms of voltage angle deviations after fault occurred. Buses 12 and 13 can be considered as the most sensitive buses in terms of voltage angle followed by buses $6,9,10,11$ and 14 . This clearly shows that voltage 
magnitude and angle are responded differently toward the fault occurences in the test system and they should be treated in more appropriate way to trade-off between the two parameters.

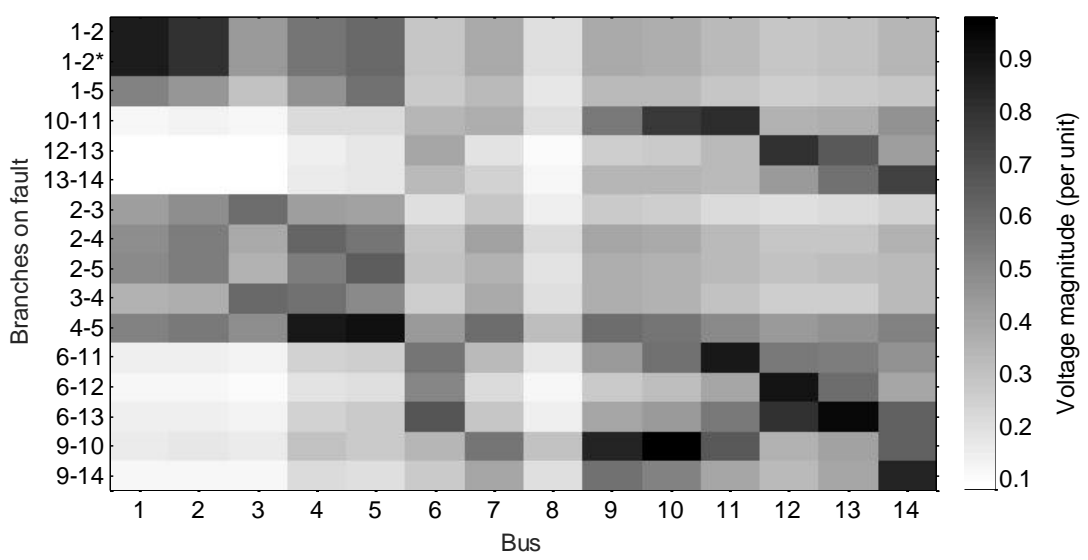

(a)

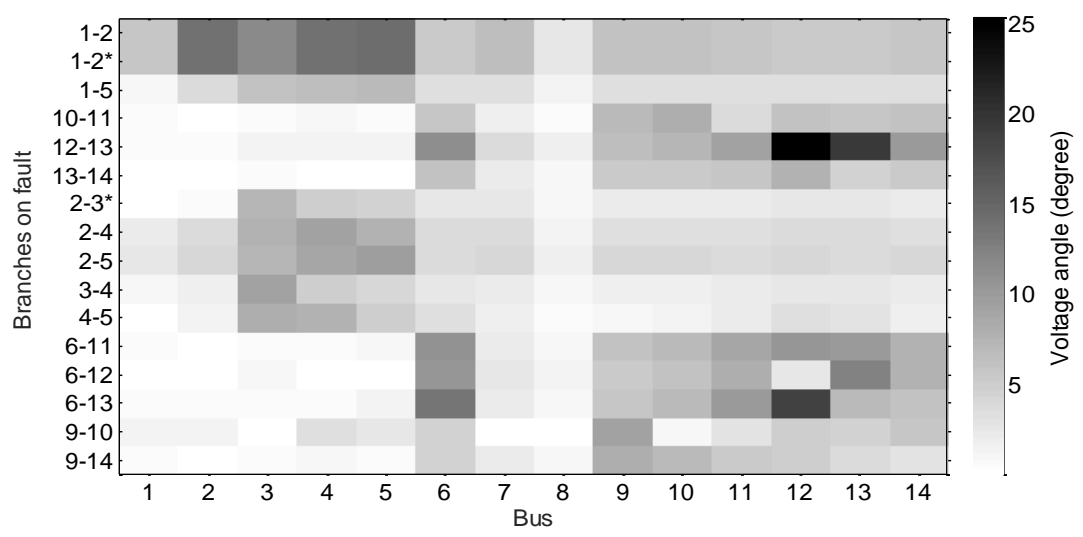

(b)

Figure 4. Sensitivity levels in each bus with respect to fault locations, (a) Voltage magnitude deviation, $|\Delta v|$, (b) Voltage angle deviation, $|\Delta \delta|$

According to $(10,11)$, the standard deviations for both indices are calculated and buses are sorted according to their sensitivity levels. The standard deviation is the most relevant statistical analysis to address an overall deviation especially for data with mixture of positive and negative deviations. Table 3 shows the standard deviations of voltage magnitude and angle that are ranked in according to their sensitivity levels. It clearly shows that bus 8 is the least sensitive and bus 12 is the most sensitive bus in terms of voltage angle. Meanwhile, bus 14 is the most sensitivite bus and bus 8 is the least sensitive in terms of voltage magnitude. Therefore, buses with higher sensitivity levels will be given more priority for the PMU placement. Table 4 shows an overall performance for the PMU placement solutions in Table 1 and they are ranked in according to the two indices from the highest to the lowest. According to the results, there are different combinations of PMU placement between the two indices. Although the combination of 2, 7, 10 and 13 is the highest based on the voltage magnitude index, it is lowest in terms of the voltage angle index. Therefore, the combination of 2, 7, 11 and 13 is selected as the best solution after considering trade-off between the indices. This is basically based on the highest rank for the voltage angle index and second highest for the voltage magnitude index. 
Table 3. Sensitivity levels of system buses based on voltage magnitude and angle deviations

\begin{tabular}{ccccc}
\hline \multirow{2}{*}{ Ranking } & \multicolumn{3}{c}{ Voltage magnitude, $\boldsymbol{v}(\mathrm{p} . \mathrm{u})$} & \multicolumn{2}{c}{ Voltage Angle, $\boldsymbol{\delta}($ degree $)$} \\
\cline { 2 - 5 } & Voltage magnitude deviation, $\sigma_{i}^{v}$ & Bus & Voltage angle deviation, $\sigma_{i}^{\delta}$ & Bus \\
\hline 1 & 1.958 & 14 & 37.317 & 12 \\
2 & 1.951 & 10 & 29.988 & 13 \\
3 & 1.912 & 12 & 27.986 & 6 \\
4 & 1.901 & 11 & 27.032 & 4 \\
5 & 1.880 & 13 & 26.615 & 5 \\
6 & 1.861 & 5 & 24.986 & 3 \\
7 & 1.797 & 4 & 23.082 & 11 \\
8 & 1.790 & 9 & 22.437 & 9 \\
9 & 1.720 & 1 & 22.207 & 14 \\
10 & 1.702 & 2 & 21.367 & 10 \\
11 & 1.520 & 6 & 21.064 & 2 \\
12 & 1.478 & 7 & 13.725 & 7 \\
13 & 1.347 & 3 & 9.118 & 1 \\
14 & 0.776 & 8 & 5.730 & 8 \\
\hline
\end{tabular}

Table 4. Performance of the feasible PMU placements in according to the proposed indices

\begin{tabular}{ccccc}
\hline \multirow{2}{*}{ Ranking } & \multicolumn{2}{c}{ Voltage magnitude, $\boldsymbol{v}(\mathrm{p} . \mathrm{u})$} & \multicolumn{2}{c}{ Voltage Angle, $\boldsymbol{\delta}$ (degree) } \\
\cline { 2 - 5 } & PMU placement (bus) & SOI $\boldsymbol{v}$ (p.u) & PMU placement (bus) & SOI $\boldsymbol{\delta}$ (degree) \\
\hline 1 & $2,7,10,13$ & 7.0128 & $2,7,11,13$ & 87.8621 \\
2 & $2,7,11,13$ & 6.9629 & $2,6,7,9$ & 85.2148 \\
3 & $2,6,7,9$ & 6.4916 & $2,8,10,13$ & 78.1512 \\
4 & $2,8,10,13$ & 6.3104 & $2,6,8,9$ & 77.2195 \\
5 & $2,6,8,9$ & 5.7892 & $2,7,10,13$ & 56.1465 \\
\hline
\end{tabular}

\section{CONCLUSION}

This paper presents new sensivity indices to select the best solution from the multiple possible solutions obtained from the exhaustive search method based on the conventional objective function to minimize the total number of PMU placement in power networks. The presence of ZIB is also possible to reduce the total number of PMU placement. The indices are constructed from fault analysis to indicate the sensitivity level of each bus toward fault occurance. A standard deviation formula is adopted in the indices to give an overall performance of each bus toward all possible fault occurance. Then, summation of the indices depending on the PMU placement is used to select the best PMU placement combination. Based on the simulation result, it shows that all buses in the network represent different sensitivity according to the fault occurance. Since higher priority will be given to more sensitive buses, the highest value of the proposed indices from the possible PMU placement combination is selected as optimal solution. In addition, the information from the installed PMU can be useful for further angle stability assessment.

\section{ACKNOWLEDGEMENTS}

This work is sponsored by Universiti Kebangsaan Malaysia under grant GUP-2018-024.

\section{REFERENCESK}

[1] F. Shi, H. Zhang and G. Xue, "Instability prediction of the inter-connected power grids based on rotor angle measurement," International Journal of Electrical Power and Energy Systems, vol. 88, pp. 21-32, 2017.

[2] A. Gourma, A. Berdai and M. Reddak, "The transient stability analysis of wind turbines interconected to grid under fault," International Journal of Electrical and Computer Engineering, vol. 10, no. 1, pp. 600-608, 2020.

[3] C. Lu, Z. Wang and Y. Yu, "Optimal PMU placement for pessimistic dynamic vulnerability assessment," IET Generation, Transmission \& Distribution, vol. 12, no. 10, pp. 2231-2237, 2018.

[4] Maveeya Baba, Nursyarizal B. M. Nor, Taib B. Ibrahim, M. Aman. Sheikh, "A comprehensive review for optimal placement of phasor measurement unit for network observability," Indonesian Journal of Electrical Engineering and Computer Science (IJEECS), vol. 19, no. 1, pp. 301-308, July 2020.

[5] A. A. Ibrahim, A. Mohamed, H. Shareef and S. P. Ghoshal, "A New Approach for Optimal Power Quality Monitor Placement in Power System Considering System Topology (Nowa metoda oceny rozmieszczenia monitorów jakości energii w systemie energetycznym z uwzględnieniem jego topologii)," Przeglad Elektrotechniczny (Electrical Review), vol. 88, no. 9A, pp. 272-276, 2012.

[6] A. A. Ibrahim, A. Mohamed and H. Shareef, "Optimal placement of power quality monitors in distribution systems using the topological monitor reach area," 2011 IEEE International Electric Machines \& Drives Conference (IEMDC), Niagara Falls, ON, pp. 394-399, 2011. 
[7] L. A. Wong, H. Shareef, A. Mohamed and A. A. Ibrahim, "Novel quantum-inspired firefly algorithm for optimal power quality monitor placement," Frontiers in Energy, vol. 8, no. 2, pp. 254-260, 2014.

[8] F. Aminifar, M. Fotuhi-Firuzabad, A. Safdarian, A. Davoudi and M. Shahidehpour, "Synchrophasor measurement technology in power systems: panorama and state-of-the-art," in IEEE Access, vol. 2, pp. 1607-1628, 2014.

[9] J. Zhao, Y. Yang and Z. Gao, "A review on on-line voltage stability monitoring indices and methods based on local phasor measurement," Dianli Xitong Zidonghua/Automation of Electric Power Systems, vol. 34, no. 20, pp. 1-6, 2010.

[10] Y. Wang, W. Li and J. Lu, "A new node voltage stability index based on local voltage phasors," Electric Power Systems Research, vol. 79, no. 1, pp. 265-271, 2009.

[11] J. Hou, C. Xie, T. Wang, Z. Yu, Y. Lü and H. Dai, "Power system transient stability assessment based on voltage phasor and convolution neural network," 2018 IEEE International Conference on Energy Internet (ICEI), Beijing, pp. 247-251, 2018.

[12] C. Shand, G. Taylor, E. Stewart, C. Roberts, A. Mcmorran and P. Mohapatra, "Improving actionable observability of large distribution networks for transmission operators to support improved system control, fault detection and mitigation," CIRED-Open Access Proceedings Journal, vol. 17, no. 1, pp. 1215-1218, Oct 2017.

[13] R. Babu and B. Bhattacharyya, "Strategic placements of PMUs for power network observability considering redundancy measurement," Measurement: Journal of the International Measurement Confederation, vol. 134, pp. 606-623, 2019.

[14] A. C. Adewole and R. Tzoneva, "Extended optimal PMU placement problem for voltage stability assessment," WSEAS Transactions on Power Systems, vol. 13, pp. 364-376, 2018.

[15] A. Biswal and H. D. Mathur, "Optimized PMU stationing for wide area monitoring of power grid," Procedia Technology, vol. 21, pp. 2-7, 2015.

[16] D. Dua, S. Dambhare, R. K. Gajbhiye and S. A. Soman, "Optimal multistage scheduling of PMU placement: an ILP approach," in IEEE Transactions on Power Delivery, vol. 23, no. 4, pp. 1812-1820, 2008.

[17] P. L. Narayana, M. Venkatesan and S. Ravindra, "Fast load voltage stability index constrained PMU placement for complete observability," International Journal of Engineering \& Technology, vol. 7, no. 4.24, pp. 20-25, 2018.

[18] M. S. Srikumar, T. Ananthapadmanbha, F. Zafar and V. Girish, "Line outage detection using phasor measurement units," Procedia Technology, vol. 21, pp. 88-95, 2015.

[19] N. H. A. Rahman and A. F. Zobaa, "Optimal PMU placement using topology transformation method in power systems,” Journal of Advanced Research, vol. 7, no. 5, pp. 625-634, 2016.

[20] A. Mahari and H. Seyedi, "Optimal PMU placement for power system observability using BICA, considering measurement redundancy," Electric Power System Research, vol. 103, pp. 78-85, Oct 2013.

[21] Q. Jiang, X. Li, B. Wang and H. Wang, "PMU-based fault location using voltage measurements in large transmission networks," in IEEE Transactions on Power Delivery, vol. 27, no. 3, pp. 1644-1652, July 2012.

[22] A. Khaleghi, M. Sadegh and M. G. Ahsaee, "Permanent fault location in distribution system using phasor measurement units (PMU) in phase domain," International Journal of Electrical and Computer Engineering (IJEECS), vol. 8, no. 5, pp. 2709-2720, October 2018.

[23] N. E. Wu, "Cost-effective upgrade of PMU networks for fault-tolerant sensing," in IEEE Transactions on Power Systems, vol. 33, no. 3, pp. 3052-3063, May 2018.

[24] H. Nazaripouya and S. Mehraeen, "Optimal PMU placement for fault observability in distributed power system by using simultaneous voltage and current measurements," 2013 IEEE Power \& Energy Society General Meeting, Vancouver, BC, pp. 1-6, 2013.

[25] Z. Jiang, S. Miao, H. Xu, P. Liu and B. Zhang, "An effective fault location technique for transmission grids using phasor measurement units," International Journal of Electrical Power and Energy Systems, vol. 42, no. 1, pp. 653-660, Nov 2012.

[26] S. Karn, S. R. Samantaray and A. Sharma, "Enhancing performance of wide-area back-up protection scheme using PMU assisted dynamic state estimator," in IEEE Transactions on Smart Grid, vol. 10, no. 5, pp. 5066-5074, Sept. 2019. 